\title{
ESPASMO HEMIFACIAL E IMPRESSÃO BASILAR ASSOCIADOS A MALFORMAÇÃO DE ARNOLD-CHIARI
}

\author{
RELATO DE CASO
}

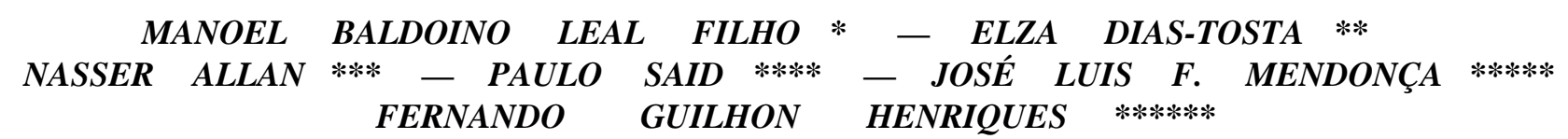

RESUMO - Os autores relatam o caso de uma paciente com espasmo hemifacial e impressão basilar associados a malformação de Arnold-Chiari. Com a descompressão cirúrgica da fossa posterior, empregada no tratamento da impressão basilar, houve melhora do quadro clínico e o espasmo hemifacial se reduziu quanto à frequência, duração e intensidade. ís enfatizada a necessidade do tratamento etiológico do espasmo hemifacial, antes de se recorrer à toxina botulínica.

PALAVRAS-CHAVE: nervo facial, espasmo, malformação de Arnold-Chiari, impressão basilar, tratamento etiológico.

Hemifacial spasm in basilar impression and Arnold- $€$ h iari malformation: Case report.

SUMMARY - The authors report a case of symptomatic basilar impression and ArnoldChiari malformation being presented as the first symptom of hemifacial spasm. The surgical treatment of the malformation resulted in inprovement of the clinical manifestation with reduction of the hemifacial spasm. The need for the aetiological therapy for the hemifacial spasm is emphasized, before symptomatic treatment with botulinum toxin is tried.

KEY WORDS: facial nerve, spasm, Arnold-Chiari malformation, basilar impression, aetiological treatment.

O espasmo da musculatura inervada pelo facial acomete um lado da face e caracteriza-se por contrações paroxísticas, irregulares e de graus variados. Iniciase geralmente pelo músculo orbiçular da pálpebra e acomete em seguida a musculatura da comissura labial ${ }^{5}>^{12}$. E mais comum na mulher, entre a quinta e sexta décadas, afeta principalmente o lado esquerdo, sendo os espasmos desencadeados pelo estresse psicológico, cansaco físico e movimentos da face, podendo ser evidenciados mesmo durante o sono e a anestesia 12. o espasmo hemifaeial (EHF) não é achado incomum na literatura médica, mas em nosso meio constitui raridade 5, principalmente quando comparado à incidência de paralisia facial periférica, outra condiçãa patológica do mesmo nervo. O EHF costumava ser considerado idiopático em sua grande maioria, mas estudos utilizando angiografia vertebral e tomografia computadorizada de crânio, evidenciaram etiologia bem definida em 83\% dos casos 3. Dentre as etiologias mais citadas encontram-se a vascular, a neoplásica, as alterações degenerativas da transição, crânio-vertebral, a inflamatória, a infecciosa e a traumática'. Existem controvérsias quanto à frequência da associação do EHF com impressão basilar (IB) e/ou malformação de Arnold-

Trabalho das Unidades de Neurologia e de Neurocirurgia do Hospital de Base do Distrito Federal: *Médico-Residente; **Neurologista, PhD; ***Neurologista; ***** «Neurocirurgião; $* * * * *$ Neurorradiologista; $* * * * * *$ Professor Adjunto, Chefe da unidade de Neurologia.

Dra. Elsa Dias-Tosta - Unidade de Neurologia, Hospital de Base do Distrito Federal - Setor Hospitalar Sul - 10000 Brasília DF - Brasil. 
Chiari (AC). Segundo ciados referidos por Brito e Silva², a associação foi encontrada em 12 de 118 casos $(10,1 \%)$, tendo em 10 destes o espasmo iniciado o quadro clínico. A associação tem sido descrita raramente na literatura nacional: três em 164 casos $^{2}$. Na Unidade de Neurologia do Hospital de Base do Distrito Federal encontraram-se apenas 1,8\%, em estudo retrospectivo de 10 anos, entre 53 casos de IB e/ou AC atendidos até 1990 (M. Moura e col.: comunicação pessoal, 1990).

O objetivo do presente relato é chamar a atenção para a necessidade de investigação cuidadosa de casos de EHF, que possibilite o tratamento etiológico.

\section{OBSERVAÇÃO}

LCR, paciente com 50 anos de idade, do sexo feminino, branca, natural da Bahia e procedente do Distrito Federal, registro 183800 do Hospital de Base de Brasília, internada em 16-abril-91. A evolução do EHF havia se dado em quatro anos. Na admissão queixava-se nos últimos) quatro meses de tontura, oscilopsia, disfagia, náusea e hipoacusia à esquerda (E). Ao exame, apresentava espasmo hemifacial à E, nistagmo horizontal bilateral rotatório e nistagmo vertical para baixo, desvio da úvula para a direita (D) e assimetria de véu, hiperestesia tátil-dolorosa na hemiface E, atrofia muscular temporal e malar E, e desvio da marcha para a D. Tinha história pregressa de lúpus eritematoso sem sinais de atividade atualmente e doença de Chagas compensada. A investigação mostrou sinais de lesão de tronco cerebral (potencial evocado), impressão basiliar (radiografia de transição crânio-cervieal) e invaginação de amigdalas cerebelares até a segunda vértebra cervical (mielografia-tomografia cervical, Fig.1)

Durante abordagem cirúrgica foi feita lamineetomia C1-C2, craniectomia occipital inferior, abertura e plástica da dura-máter com faseia lata. Após um mês já apresentava redução na frequência, duração e intensidade dos espasmos, avaliados segundo os critérios preconizados por Marti e cols. 7, diminuição da tontuna e desaparecimento da oscilopsia, disfagia e da náusea. Ao exame, permanecia o desvio da úvula para a D, o reflexo nauseoso estava abolido, enquanto a sensibilidade tátil-dolorosa da face e a marcha haviam se normalizado.
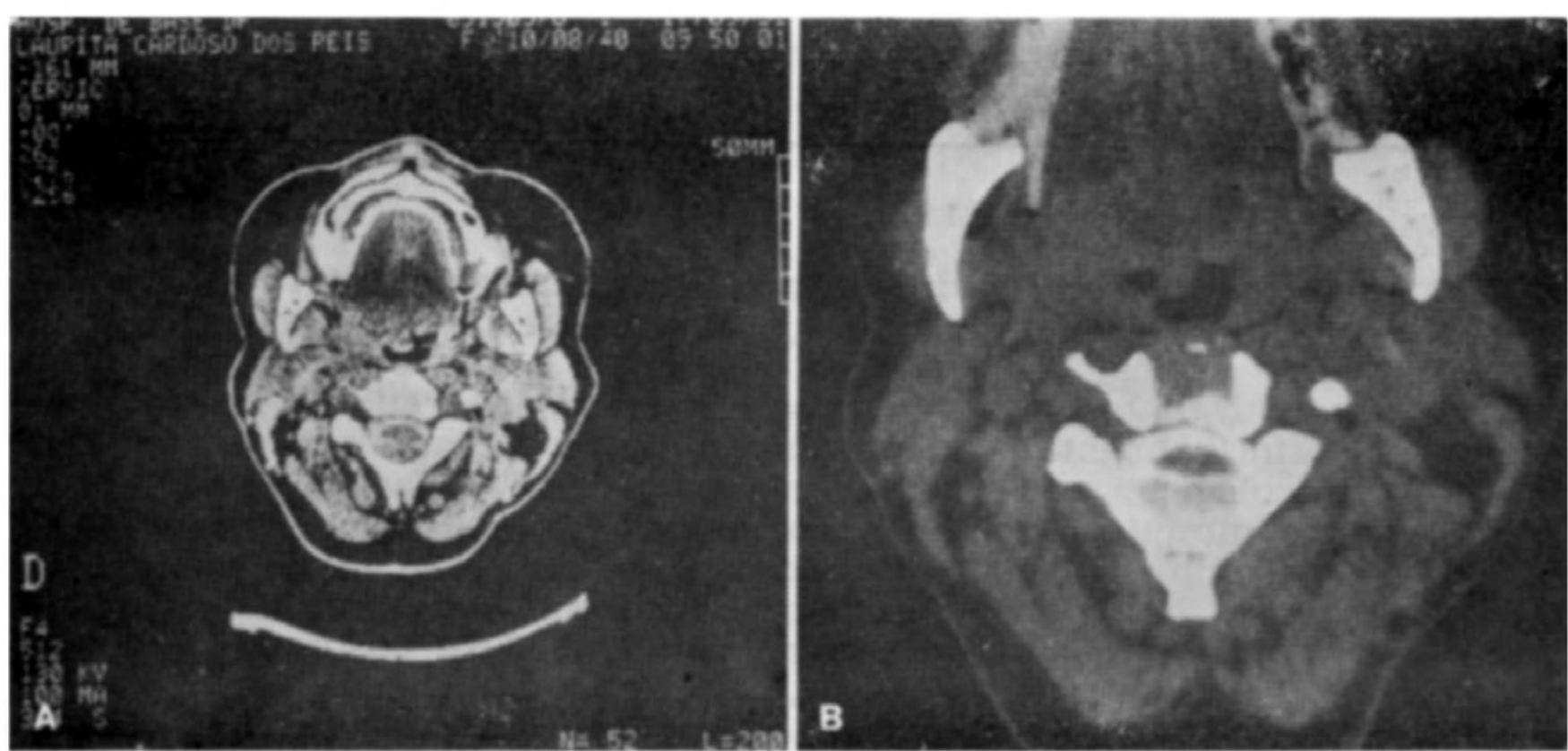

Fig. 1. Caso LCR. (A) Mielografia com tomografia computadorizada da região cervical evidenciando herniação das amigdalas cerebelares até C2. (B) Detalhe da herniação.

\section{COMENTÁRIOS}

Qualquer que seja a etiologia encontrada para o EHF, a topografia do sofrimento neural estará na porção intracraniana e extra-axial do faciais, o mecanismo fisiopatológico é ainda motivo de discussão sendo postulado que a compressão constante, ainda que discreta, do nervo facial resulte em desmielinização 
focal com formação de falsas sinapses neste local ${ }^{14}$. Outros autores admitem que possa existir estado de hiperexcitabilidade do núcleo do facial em resposta a estímulos periféricos, ainda que continuem aceitando a desmielinização focal do nervo como ponto de transmissão transaxônica 8,17 e que os dois mecanismos fỉsiopatológicos não sejam excludentes s,n. Além da compressão do facial por vasos ectasiados e tortuosos, podem também dar origem ao EHF a distensão do nervo e a diminuição do fluxo sanguíneo no tronco cerebral ${ }^{2}$, o que se supõe que ocorra na IB. Esta, que é uma malformação óssea do crânio, se associa com frequência a outras malformações ósseas e do sistema nervoso e à presença de vasos anômalos com trajetos aberrantes ${ }^{2}>^{3}$ que podem participar da gênese do EHF. As manifestações clínicas mais comuns da malformação de Ârnold-Chiari decorrem de desordens bulbo-medulares, cerebelares, obstruçåo do IV ventrículo e siringomielia associada 15 e apresentam-se, em ordem de frequência, com dor, fraqueza, dormência e desequilíbrio 13 .

Os sinais preditivos de evolução desfavorável, apesar do tratamento cirúrgico, são atrofia muscular, duração dos sintomas superior a 24 meses, ataxia, nistagmo, hiperestesia trigeminal e escoliose $e^{4}$.

A mielografia costuma ser o exame mais útil^^ a tomografia computadorizada com contraste pode evidenciar alterações no sistema vértebro-basilar 3 Ultimamente a ressonância magnética vem trazendo importante subsídio ao diagnóstico dc lesões de fossa posterior mas, no presente relato, o exame definitivo foi a mielografia com tomografia computadorizada. $O$ estudo neurofisiológico, indicando sinais de comprometimento do tronco cerebral, motivou a investigação da fossa posterior com mais empenho.

A descompressão cirúrgica da região do forame magno leva a bons resultados Lio, principalmente quando não existe associação com siringomielia i. Muitas vezes ocorrem aderências da aracnóide e siringomielia 13 , requerendo nestes casos um complemento à técnica cirúrgica adotada previamente.

$\hat{\mathbf{E}}$ necessário que o tratamento da causa básica reverta, pelo menos parcialmente, o quadro clínico e que a proposta terapêutica não seja feita tardiamente relacionados à evolução desfavorável (atrofia muscular, ataxia, nistagmo cirúrgico da impressão basilar e/ou Arnold-Chiari nem sempre resulta em desaparecimento do EHF 2. No presente relato foram notados alguns sinais supostamente relacionados à evolução desfavorável (atrofia muscular, ataxia, nistagmo e hiperestesia trigeminal). No entanto, a descompressão cirúrgica trouxe inegável benefício à paciente. Para o EHF que ainda restar, planeja-se a aplicação de toxina butulínica\&.H após um seguimento de 12 meses, prazo necessário para se dar remielirüzação do nervo, segundo medidas dè latência realizadas vários meses após a descompressão io.

\section{REFERENCIAS}

1. Banerjj NK, Millar JHD. Chiari malformation presenting in adult life: its relationship to syringomyelia. Brain 1974, 97:157-168.

8. Brito JCF, Silva JAG. Espasma hemifacial na impressão basiliar: registro de três casos operados. Arq Bras Neurocirurg 1983, 2:125-132.

3. Digre KB, Corbett JJ, Smoker WRK, MkKusker S. CT and hemifacial spasm. Neurology 1988, 38:1111-1113.

4. Dyste GN, Menezes AH, Vangilder JC. Symptomatic Chiari malformations: an analysis of presentation, management, and long-term outcome. J Neurosurg 1989, 71:159-168.

5. Fortes-Rego J. Espasmo hemifacial: registro de um caso. Arq Neuro-Psiquiat (São Paulo) 1985, 43:98-101.

6. Jankovic J, Schwartz K, Donovan DT. Botulinum toxin treatment of cranial-cervical dystonia, spasmodic dysphonia, other focal dystonias and hemifacial spasm. J Neurol Neurosurg Psychiatry 1990, 53:633-639.

7. Martí MJ, Tolosa E, Alom J. Botulinum toxin in hemifacial spasm: a double-blind controlled trial. In: Bartko D. (ed): New Trends in Clinical Neuropharmacology. London: John Libbey, 1988, p 304307.

8. Moller R. Hemifacial spasm ephaptic transmission or hyperexcitability of the facial motor nucleus? Exp Neurol 1987, 98:110-119. 
9. Moller AR, Jannetta PJ. Hemifacial spasm: results of electrophysiologic recording during microvascular decompression operations. Neurology 1985, 35:969-974.

10. Montoya A, Acevedo CA. Decompresión mocroquirúrgica en la fosa posterior para tratamiento del espasmo hemifacial. Colombia Medida 1984, 15:30-32.

11. Nielsen VK. Indirect and direct evidence of ephaptic transmission in hemifacial spasm. Neurology 1986, 36:592-593.

12. Niemeyer P Filho, Bezerra M, Mufarrej G. Espasmo hemifacial: resultado da descom pressão microvascular em 53 pacientes. Arq Neuro-Psiquiat (São Paulo) 1990, 48:210-216.

13. Paul KS, Lye RH, Strang FA, Dutton J. Arnold-Chiari malformation: review of 71 cases. J Neurosurg 1983, 58:183-187.

14. Ravits J, Hallet M. Pathophisiology of hemifacial spasm. Neurology 1986, 36:591-593.

15. Taylor AR, Chfakravorty BC. Clinical syndromes associated with basilar impression. Arch Neurol 1964, 10:475-485.

16. Tolosa E, Marti MJ, Kulisevsky J. Botulinum toxin injection therapy for hemifacial spasm. Adv Neurol 1988, 40:479-491.

17. Wilkins RH. Hemifacial spasm. Contemporary Neurosurgery 1991, 13:1-5. 